\title{
A NOTE ON \\ FINITE-DIMENSIONAL DIFFERENTIABLE MAPPINGS
}

\author{
K. J. PALMER and SADAYUKI YAMAMURO
}

(Received 24 August 1967, revised 14 December 1967)

Let $E$ be a real infinite-dimensional Banach space. Let $\mathscr{L}$ be the Banach algebra of all continuous linear mappings of $E$ into itself with the topology defined by the norm:

$$
\|l\|=\sup _{\|x\|=1}\|l(x)\| \quad(l \in \mathscr{L}) .
$$

A mapping $t$ of $E$ into itself is said to be (Fréchet)-differentiable if, for each $a \in E$, there exists an $l \in \mathscr{L}$ such that

$$
\lim _{\|x\| \rightarrow 0} \frac{1}{\|x\|}\|f(a+x)-f(a)-l(x)\|=0 .
$$

The linear mapping $l$ is determined uniquely for each $a$. We denote it by $f^{\prime}(a)$ and call it the derivative of $f$ at $a$. The set of all differentiable mappings of $E$ into itself is denoted by $\mathscr{D}$. Following definitions were given in [2].

and

$$
\begin{aligned}
d(f) & =\left\{f^{\prime}(x) \mid x \in E\right\} & & \text { for } f \in \mathscr{D}, \\
d(M) & =\bigcup_{f \in M} d(f) & & \text { for } M \subset \mathscr{D}, \\
d^{-1}(N) & =\{f \in \mathscr{D} \mid d(f) \subset N\} & & \text { for } N \subset \mathscr{L} .
\end{aligned}
$$

A mapping $f$ is said to be finite-dimensional if the range $R(f)$ is contained in a finite-dimensional subspace of $E$. The set of all finite-dimensional mappings of $E$ into itself is denoted by $\mathscr{F}$.

The purpose of this paper is to show that

$$
\mathscr{F} \cap \mathscr{D} \varsubsetneqq d^{-1}(\mathscr{F} \cap \mathscr{L})
$$

and

(2) $f \in \mathscr{F} \cap \mathscr{D}$ if and only if there exists a finite-dimensional subspace $E_{0}$ of $E$ such that $\bigcup_{x \in E} R\left(f^{\prime}(x)\right) \subset E_{0}$. 


\section{Proof of (1)}

Let $f$ be in $\mathscr{F} \cap \mathscr{D}$. Since $f \in \mathscr{F}$, there exists a finite-dimensional subspace $E_{0}$ of $E$ such that $R(f) \subset E_{0}$. Therefore, from

$$
f^{\prime}(a)(x)=\lim _{\varepsilon \rightarrow 0} \frac{1}{\varepsilon}[f(a+\varepsilon x)-f(a)]
$$

it follows that $R\left(f^{\prime}(a)\right) \subset E_{0}$, which means that $f \in d^{-1}(\mathscr{F} \cap \mathscr{L})$.

To show that the equality does not hold, we consider the case when $E=L^{2}[-\pi, \pi]$, the Banach space of all square-integrable measurable real functions on the interval $[-\pi, \pi]$. Then, the mapping $f$ :

$$
f(x)(t)=\sin (x(t))
$$

defined for all $x \in L^{2}[-\pi, \pi]$. The fact that $f \in \mathscr{D}$ and

$$
f^{\prime}(a)(x)(t)=\cos (a(t)) x(t)
$$

follows from Theorem 3.3 and Theorem 20.2 of [1]. Next, take the function $e(t)$ such that

$$
e(t)=t \text { for } t \in[-\pi, \pi],
$$

and consider the one-dimensional linear mapping

$$
l(x)=(x, e) e,
$$

where $(x, e)$ denotes the inner product of $x$ and $e$. Then, for the mapping

we have

$$
g(x)=f(l(x)),
$$

$$
g^{\prime}(a)(x)=f^{\prime}(l(a)) l(x),
$$

from which it follows that

$$
\begin{aligned}
R\left(g^{\prime}(a)\right) & =\left\{f^{\prime}(l(a))(x, e) e \mid x \in E\right\} \\
& =\left\{\xi f^{\prime}(l(a)) e \mid-\infty<\xi<\infty\right\},
\end{aligned}
$$

which is obviously a one-dimensional subspace of $E$.

On the other hand, $R(g)$ is not finite-dimensional, because

$$
g\left(n \frac{e}{\|e\|^{2}}\right)(t)=f(n e)(t)=\sin (n e(t))=\sin n t
$$

for $n=1,2, \cdots$ and $\{\sin n t\}$ is an orthogonal system of $L^{2}[-\pi, \pi]$. 


\section{Proof of (2)}

We assume that $f \in \mathscr{D}$ and $\bigcup_{x \in E} R\left(f^{\prime}(x)\right) \subset E_{0}$ for some finite-dimensional subspace $E_{0}$. Take an arbitrary $\bar{x} \in \bar{E}$ (the conjugate space of $E$ ) such that $\bar{x}(y)=0$ for $y \in E_{0}$. Then, by Lemma 3.2 of [1], we have, for every $x \in E$,

$$
\bar{x}(f(x)-f(0))=\bar{x}\left(f^{\prime}(\tau x)(x)\right) \text { for some number } \tau,
$$

from which it follows that

This means that the set

$$
\bar{x}(f(x)-f(0))=0 .
$$

$$
\{f(x)-f(0) \mid x \in E\}
$$

is contained in $E_{0}$. Therefore, $R(f)$ is contained in a finite-dimensional subspace that is generated by $E_{0}$ and $f(0)$. The other half was proved in the previous section.

\section{Remark}

As in [2], let us regard $\mathscr{D}$ as a near-ring. The example given in the section 1 shows that $\mathscr{F} \cap \mathscr{D}$ is not an ideal of $\mathscr{D}$. (We only consider two-sided ideals.) On the other hand, by the fact proved in [2], $d^{-1}(\mathscr{F} \cap \mathscr{L})$ is a $d$-ideal of $\mathscr{D}$. (An ideal $I$ of the near-ring $\mathscr{D}$ is said to be a $d$-ideal if $d^{-1} d(I)=I$.) Moreover, we can prove that

$$
d^{-1}(\mathscr{F} \cap \mathscr{L}) \text { is the second smallest d-ideal of } \mathscr{D},
$$

and

(4) $\quad d^{-1}(\mathscr{F} \cap \mathscr{L})$ is the smallest among d-ideals $I$ such that $d(I)$ is not the zero-ideal of the Banach algebra $\mathscr{L}$.

Proof. It has been shown in [2] that the set $I(E)$ of all constant mappings is the smallest $d$-ideal of $\mathscr{D}$ and $d(I(E))=(0)$. Let us take an arbitrary $d$-ideal $I$. If $d(I)=(0)$, we have

$$
I=d^{-1} d(I)=d^{-1}((0))=I(E) .
$$

If $d(I) \neq(0)$, since $d(I)$ is a non-zero-ideal of $\mathscr{L}$, we have $d(I) \supset \mathscr{F} \cap \mathscr{L}$, from which it follows that

$$
I=d^{-1} d(I) \supset d^{-1}(\mathscr{F} \cap \mathscr{L}) .
$$




\section{References}

[1] M. M. Vainberg, Variational methods for the study of non-linear operators, translated by A. Feinstein. (Holden-Day, Inc. 1964).

[2] Sadayuki Yamamuro, 'A note on d-ideals in some near-algebras', Journ. Australian Math. Soc., 7 (1967), $129-134$.

Australian National University 